\section{SAT0362 PERFORMANCE OF THE 2015 ACR/EULAR CLASSIFICATION CRITERIA FOR GOUT IN KOREAN PATIENTS PRESENTING WITH ACUTE ARTHRITIS}

I.A. Choi ${ }^{1}$, Y.-J. Ha ${ }^{2}$, E.H. Kang ${ }^{2}$, Y.J. Lee ${ }^{2}$, K. Shin ${ }^{3}$, E.Y. Lee ${ }^{4}$, E.B. Lee ${ }^{4}$, Y. W. Song ${ }^{4}{ }^{1}$ Division of Rheumatology, Department of Internal Medicine, Chungbuk National University Hospital, Cheongju; ${ }^{2}$ Division of Rheumatology, Department of Internal Medicine, Seoul National University Bundang Hospital, Seongnam; ${ }^{3}$ Division of Rheumatology, Department of Internal Medicine, SMG-SNU Boramae Medical Center, ${ }^{4}$ Division of Rheumatology, Department of Internal Medicine, Seoul National University Hospital, Seoul, Korea, Republic Of

Background: A definite diagnosis of gout requires the detection of monosodium urate (MSU) crystals in synovial fluid or tissue. However, the procedure involved is invasive and often not available in acute or primary care settings. Therefore, several alternate sets of classification criteria for gout have been developed.

Objectives: This study was undertaken to assess the performance of the 2015 American College of rheumatology (ACR)/European League Against Rheumatism (EULAR) classification criteria for gout and to compare its performance to previous sets of criteria including the New York, American Rheumatism Association (ARA) 1977 and Mexico criteria, in Korean patients presenting with acute arthritis.

Methods: Patients with acute arthritis who attended rheumatology clinics and underwent arthrocentesis were enrolled in the study, during February 2017 and December 2017. The gout group included patients with MSU crystals in synovial fluid or tissue, as assessed by an experienced rheumatologist or pathologist. Since the presence of MSU crystals alone is sufficient to fulfil the criterion for gout according to the 2015 ACR/EULAR classification, New York, ARA 1977 and Mexico criteria, this criterion is considered to have a $100 \%$ sensitivity in the gout group by our definition. Therefore, we excluded positive MSU crystal results in these four criteria sets to avoid a case of circular reasoning.

Results: A total of 116 gout patients (as determined by the presence of MSU crystals) and 71 non-gout patients participated in the study. The number of male patients in the gout group $(94.8 \%)$ was higher than that in non-gout group $(15.1 \%)$. The mean $( \pm S D)$ age of the patients was $58.4( \pm 15.0)$ and $64.5( \pm 13.3)$ years in the gout and non-gout group, respectively.

The sensitivity, specificity, positive predictive value and negative predictive value of the criteria sets were $87.1 \%, 100 \%, 100 \%$ and $73.2 \%$ respectively (area under the curve, AUC 0.975) for the 2015 ACR/EULAR classification criteria, 71.5\%, $97.2 \%, 97.6 \%$ and $67.6 \%$ (AUC 0.960 ) for the clinical-parameters-only version of the 2015 ACR/EULAR classification criteria, $68.1 \%, 81.7 \%, 85.9 \%$ and $61.0 \%$ (AUC 0.784) for the New York criteria, 77.6\%, 81.7\%, 87.4\% and 69.0\% (AUC 0.878 ) for the ARA 1977 criteria, and $87.9 \%, 63.4 \%, 79.7 \%$ and $76.3 \%$ (AUC 0.878) for the Mexico criteria.

To determine the performance of the existing criteria sets for gout without relying on the detection of MSU crystals, we also calculated the performance of the criteria assuming an absence of both positive and negative crystal test results. By this parameter, the sensitivity of the 2015 ACR/EULAR classification criteria was $77.6 \%$, specificity was $84.8 \%$, positive predictive value was $89.1 \%$, and negative predictive value was $69.8 \%$ (AUC 0.918). The performance of the New York, ARA 1977 and Mexico criteria did not change.

Conclusions: The performance of the 2015 ACR/EULAR classification criteria for gout was better than that for the New York, ARA 1977 and Mexico criteria in Korean patients presenting with acute arthritis. When an MSU crystal identification test is not available, 2015 ACR/EULAR classification criteria for gout could be a useful option in the diagnosis of gout in Korean patients presenting with acute arthritis.

Disclosure of Interest: None declared

DOI: 10.1136/annrheumdis-2018-eular.2171

\section{SAT0363 ARE “TARGET-URATE” AND REMISSION CRITERIA POSSIBLE IN SEVERE GOUT? A REAL LIFE EXPERIENCE IN A COHORT}

J. Vazquez-Mellado ${ }^{1}$, C. O. Lopez-Lopez ${ }^{2}$, E. Alvarez-Hernandez ${ }^{1}$, I. PelaezBallestas $^{1}$, C. Gomez-Ruiz ${ }^{1}$, A. Vazquez-Mellado ${ }^{1}$, R. Burgos-Vargas ${ }^{1}$ on behalf of GRESGO cohort. ${ }^{1}$ Rheumatology, ${ }^{2}$ Rehabilitation and physical therapy, Hospital General De México, México, Mexico

Background: Gout remission, quality of life and disability improvement are the main objectives of chronic treatment. Gout T2T goal is to achieve and maintain target urate $<6 \mathrm{mg} / \mathrm{dL}$ and $<5 \mathrm{mg} / \mathrm{dL}$ (TU) in severe gout (SG).

Objectives: To determine in a cohort of gout patients, SG frequency, associated factors, percentage of patients achieving TU and remission criteria.

Methods: Patients with gout (ACR-EULAR) from GRESGO cohort were included and evaluated at baseline and bi-annually. At baseline visit, information about gout, life style changes and treatment were discussed with the patients and relatives. Prescription for gout and associated diseases was according to published guides and available drugs. Measures: demographic, clinical and biochemical. HAQ, EUROQoL, VAS-pain and global health. SG: $\geq 5$ tophi and/or intradermal tophi. TU:s $U A<6 \mathrm{mg} / \mathrm{dL}$ and $<5 \mathrm{mg} / \mathrm{dL}$; remission according to published proposal (de Lautour H/2016). This protocol was approved by the local IRB, all patients signed informed consent. Statistical analysis: $t$ test, $\mathrm{X} 2$, Friedman, multiple-correlation and multivariable analysis.

Results: At baseline visit 500 gout patients were included, (221/44\% with SG and 279/56\% non-SG). Males $97 \%$, all received individualised ULT treatment (allopurinol and/or probenecid). The whole group, improved significantly during follow-up in urate, flares, tophus burden, HAQ, pain, EUROQoL and renal function. SG patients were significantly: younger at onset, with lower educational level, socioeconomic status, more laboral absenteeism. Also, had longer disease duration and significant differences in all clinical variables related to severity:>joint count, >index tophi size, $>\%$ lithiasis, $>$ HAQ score, $>$ EUROQoL and $<M D R D$, all these outcomes improved significantly during follow-up. In multivariate analysis, disease duration (p0.008) and flares/years (p0.055) persist significantly associated to $S G$ at baseline. SG patients received significantly higher allopurionol doses (>600 mg/day) and required more frequently glucocorticoids. Eleven patients died, 1 year after baseline $~ 50 \%$ patients were lost. Among those that continued, target urate $<6 \mathrm{mg} / \mathrm{dL}$ was possible in only $50 \%-70 \%$ of the patients followed for $3-5$ years. Less than $50 \%$ of patients achieved $\mathrm{TU}<5 \mathrm{mg} / \mathrm{dL}$ during long follow up. There were no significant differences in most percentages of TU 6 or $5 \mathrm{mg} / \mathrm{dL}$ between SG VS non-SG patients (see table 1). Although, both groups improved significantly in all clinical outcomes. Remission definition require none tophi and at least 12 months follow-up, it was observed in $9.4 \%$ of non-SG patients.

Percentage of patients achieved TU: ("significant differences)

$\mathrm{TU}<6 \mathrm{mg} / \mathrm{dL}<5 \mathrm{mg} / \mathrm{dL}$

\begin{tabular}{lccccc}
\hline & $\begin{array}{c}\text { SG } \\
\%\end{array}$ & $\begin{array}{c}\text { Non- } \\
\text { SG } \\
\%\end{array}$ & $\begin{array}{c}\text { SG } \\
\%\end{array}$ & $\begin{array}{c}\text { Non- } \\
\text { SG } \\
\%\end{array}$ & N \\
\hline Baseline & 12 & 16 & 1 & 8 & 500 \\
6 & 21 & 24 & 11 & 15 & 329 \\
12 & 35 & 44 & 16 & 22 & 254 \\
18 & 53 & 55 & 28 & 26 & 208 \\
24 & 58 & 52 & $37^{*}$ & 24 & 189 \\
30 & 56 & 51 & 32 & 28 & 152 \\
36 & 58 & 59 & 38 & 34 & 131 \\
42 & 70 & 58 & 38 & 30 & 94 \\
48 & $73^{*}$ & 50 & 35 & 27 & 81 \\
54 & 63 & 54 & 0 & 0 & 53 \\
60 & 53 & 43 & 42 & 19 & 40 \\
\hline
\end{tabular}

Conclusions: In our population, SG is very frequent and associated with younge age at onset, low socioeconomic and educational level, longer duration and severe disease. Although they receive more intense treatment according to availability, TU and remission criteria are seldom observed and very difficult to achieve. Earlier diagnosis, intensive and adequate treatment should change these results. Disclosure of Interest: None declared

DOI: 10.1136/annrheumdis-2018-eular.1730

\section{SAT0364 HIGH PREVALENCE OF ENTHESOPATHIES IN PATIENTS WITH X-LINKED HYPOPHOSPHATEMIA}

A. Salcion Picaud ${ }^{1}$, L. Lassalle ${ }^{1}$, V. Merzoug ${ }^{2}$, A. Usardi ${ }^{2}$, A. Rothenbuhler $^{2}$, P. Kamenicky' ${ }^{2}$ C. Roux ${ }^{1}$, A. Linglart ${ }^{2}$, K. Briot ${ }^{1} .{ }^{1}$ Cochin Hospital, Paris; ${ }^{2}$ Kremlin Bicêtre Hospital, Le Kremlin Bicêtre, France

Background: X-linked hypophosphatemia (XLH) is a rare genetic disease (incidence $1 / 20000$ ) due to mutations in the PHEX gene. The genetic defects is associated with high level of FGF23 leading to impaired renal phosphate reabsorption and $1,25(\mathrm{OH})$ vitaminD synthesis. Most patients are diagnosed at walking age because of lower limb deformities and rickets. In adulthood, patients complain of musculoskeletal pain in relation to bone lesions (pseudofractures, osteomalacia) and joint involvement but also ossification of entheses. The latter is a strong determinant of the altered quality of life in this population.

Objectives: The aim of this work was to describe the structural bone and joint involvement of the entire skeleton in a large cohort of adult patients with XLH.

Methods: The study was prospectively conducted in symptomatic adult XLH patients in the context of the systematic evaluation at the French reference centre for rare diseases of calcium and phosphate metabolism. 81 of the 136 patients included between June 2011 and December 2017 had full body x-rays performed with the EOS low-radiation system. Two trained readers performed a standardised analysis of radiographs with a reading grid including the collection of ossifications of the anterior and lateral vertebral ligament, iliac crests, ischial sites, cotyles and achilles tendons; osteophytes on the spine and hip osteoarthritis; and sacroiliac joint aspect.

Results: Of the 81 patients, 55 are women (68\%), mean age $42.4 \pm 13.6$ years diagnosed on average at the age of 11 years. $63(78 \%)$ patients were treated during childhood with phosphate and/or vitamin D supplementation. At the time of study, $41(50.6 \%)$ were still upon phosphate supplements and/or vitamin D 